Original Paper http://ajol.info/index.php/ijbcs http://indexmedicus.afro.who.int

\title{
Caractérisation de la végétation ligneuse du bassin versant de la Maggia dans la commune rurale de Bagaroua (région de Tahoua)
}

\author{
Amadou GARBA $^{1 *}$, Idrissou Tahirou DJIMA ${ }^{1}$, Lawali ABDOU ${ }^{2}$ et Ali MAHAMANE ${ }^{1,3}$ \\ ${ }^{1}$ Université Abdou Moumouni, Faculté des Sciences et Techniques, \\ Département de Biologie, Laboratoire Garba Mounkaila, BP 10662, Niamey, Niger. \\ ${ }^{2}$ Université de Diffa, Faculté des Sciences Agronomiques, BP 78, Diffa, Niger. \\ ${ }^{3}$ Laboratoire Garba Mounkaila, Département de Biologie, Faculté des Sciences et Techniques, \\ Université Abdou Moumouni de Niamey, Niger. \\ *Auteur correspondant ; E-mail : djimaidrissou2007@yahoo.fr; garbaamadou10@yahoo.fr \\ Tel (+227) 99422021
}

\section{RESUME}

Le bassin versant de la Maggia est située dans la région de Tahoua. La présente étude vise à caractériser la végétation ligneuse du bassin versant sur le plan de sa composition floristique et de sa structure. Les données ont été collectées au moyen des relevés floristiques, de mesures de diamètres à 1,30 m pour les arbres et $0,5 \mathrm{~m}$ pour les individus ayant un diamètre inférieur à $5 \mathrm{~m}$. La richesse spécifique de ce bassin versant est de 22 espèces réparties en 10 familles et 18 genres dont les plus importants sont les LeguminoseaeMimosoideae et les Combretaceae. L'analyse des structures en diamètre et en hauteur montre que le peuplement ligneux de cette zone est dominé par des individus jeunes, les individus âgés sont très rares. En ce qui est des types biologiques, la flore du bassin versant de la Maggia est dominée par des microphanérophytes suivis des nanophanerophytes. Pour les types phytogéographiques ce sont les espèces Soudano-Zambéziennes (SZ) suivis des espèces Soudano-Zambéziennes-Saharo-Sindiènnes (SZ-Sah.S) qui sont dominantes dans cette zone ensuite viennent les Guinéo-Congolaises-Soudano-Zambéziennes-Saharo-Sindienne ((GC-SZ-Sah.S).

(C) 2017 International Formulae Group. All rights reserved.

Mots clés : Végétation ligneuse, formes biologiques, types phytogéographique, bassin versant, vallée.

\section{Characterisation of the woody vegetation of the Maggia watershed in rural commune of Bagaroua (Tahoua region)}

\begin{abstract}
ABSTRAT
The Maggia watershed is located in the Tahoua region. The purpose of this study is to characterize the woody vegetation of the watershed in terms of its floristic composition and structure. Data were collected from floristic surveys, diameter measurements of $1.30 \mathrm{~m}$ for trees and $0.5 \mathrm{~m}$ for individuals with diameters less than $5 \mathrm{~m}$. The specific richness of this watershed consists of 22 species divided into 10 families and 18 types of which the most important are Leguminoseae-Mimosoideae and Combretaceae. The analysis of the structures in terms of diameter and height shows that the woody population of this area is dominated by young individuals,
\end{abstract}


elderly individuals are very rare. Regarding the biological types, the flora of the watershed of the Maggia is dominated by microphanerophytes followed by nanophanerophytes. For the phytogeographical types, we are the Sudano-Zambezian species (SZ), followAed by those of the Sudano-Zambezian-Saharan-Sindian species (SZ-Sah.S) which are dominant in this zone. Then comes the Guineo-Congolese-Sudanese-Zambezian -Saharo Sindian (GC-SZ-Sah.S).

(C) 2017 International Formulae Group. All rights reserved.

Keywords: Woody vegetation, biological forms, phytogeographic type, watershed, valley.

\section{INTRODUCTION}

Au sahel, particulièrement au Niger, les $3 / 4$ de la population estimée à 17.129.076 habitants vivent des activités agricoles. La plupart de ces activités qui dépendent intégralement des conditions climatiques, sont confrontées de nos jours aux problèmes des aléas climatiques entraînant une baisse drastique de la productivité des terres. Cette situation, tout en créant un déséquilibre entre la production alimentaire des terres et la population qui croît de façon exponentielle, maintient le pays dans une situation d'insécurité alimentaire chronique et dans une forte dépendance des aides extérieures. Ainsi, pour y faire face, le Niger s'est engagé dans la lutte contre la pauvreté, en adoptant, après un processus participatif, en janvier 2002, la Stratégie de Réduction de Pauvreté (SRP). Cette stratégie, qui constitue le seul cadre de référence pour le développement économique et social pour le Niger, fait du secteur rural le moteur de croissance économique. Pour permettre à ce secteur de jouer pleinement ce rôle, le Gouvernement a élaboré et adopté en novembre 2003, la Stratégie de Développement Rural (SDR) dont l'objectif général est de réduire l'incidence de la pauvreté en milieu rural de $66 \%$ à $52 \%$ à l'horizon 2015. Compte tenu de la prédominance de l'agriculture dans le secteur rural, de la précarité climatique et du potentiel irrigable, l'effort du développement agricole pour l'atteinte de cet objectif est orienté vers les cultures irriguées à travers la maitrise des eaux de surface et une meilleure mobilisation des eaux souterraines. Cette situation d'opportunité a fait l'objet de l'intervention de plusieurs projets de développement dans ce secteur dont celui du projet de développement des exportations et des marchés agro-sylvopastoraux (PRODEX) pour la réalisation des seuils d'épandage dans certaines vallées comme celle de la Maggia, afin de soutenir les producteurs confrontés à un problème de rabattement précoce des nappes. Le bassin versant de la Maggia est un écosystème qui contribue de manière significative à la résilience des populations de cette localité de la région de Tahoua. En effet, pendant la période de décrue, les populations exploitent la vallée du bassin versant en pratiquant plusieurs types des cultures de contre saison dont la principale reste la culture de dolique qui est une espèce de haricot très riche en protéine. Dans ce bassin versant, la végétation ligneuse assure l'équilibre de l'écosystème en protégeant la vallée contre l'érosion hydrique et éolienne, ce qui permet à la nappe phréatique de pouvoir se recharger. Aujourd'hui, avec les sécheresses répétitives liées à la rareté des pluies au sahel, la coupe abusive des arbres et le surpâturage, on assiste à un rebattement précoce de la nappe lié au phénomène d'ensablement créant ainsi un déséquilibre au sein de l'écosystème. Cet ensablement a conduit à une dégradation de la vallée de la Maggia caractérisée par une baisse de la production et une diminution de l'espace cultivable dans la vallée. Les populations n'arrivent plus à pratiquer la 
culture de dolique que dans des espaces isolés de la vallée où la nappe peut de se recharger et cette situation engendre parfois des conflits entre les exploitants de la vallée. Pour permettre à l'écosystème du bassin versant de la Maggia de trouver son équilibre, il est nécessaire de sauvegarder la biodiversité végétale ligneuse qui protège la vallée contre l'ensablement. La gestion durable de ces ressources forestières passe nécessairement par une meilleure connaissance de leur diversité. Un inventaire de cette diversité permettra de mieux connaître ses éléments constitutifs et d'effectuer un suivi de sa dynamique au cours du temps (Larwanou, 2005). La présente étude s'inscrit dans ce cadre et a pour objectif de caractériser la végétation ligneuse du bassin versant.

De manière spécifique, il s'agit d'inventorier les espèces végétales ligneuses, de déterminer les types biologiques et phytogéographiques ainsi que les structures en diamètre et en hauteur du peuplement.

\section{MATERIEL ET METHODES}

\section{Zone d'étude}

La présente étude a été réalisée au niveau du site de Gougouhema (village) situé à la latitude $14,2068^{\circ}$ Nord et la longitude 04 , $8000^{\circ}$ Est et le site de Libatan (village) situé à la Latitude 14, $356930^{\circ}$ Nord et Longitude $04,993640^{\circ}$ Est. Ces deux villages sont tous situés dans la commune de Bagaroua. La commune est située dans la région de Tahoua. La région de Tahoua est localisée dans la partie Nord du pays. Elle est créée en 1998 par la loi $n^{\circ}$ 98- 31 du 14 septembre 1998. Elle est limitée au Nord par la région d'Agadez, au Sud par la République Fédérale du Nigeria, à l'Est par la région de Maradi et à l'Ouest par la région de Dosso et de Tillabéry et la République du Mal. Elle couvre une superficie de $113371 \mathrm{Km}^{2}$ soit 8,95\% du territoire national. La région est caractérisée par deux saisons distinctement séparées. La saison des pluies entre mai et septembre est relativement courte en comparaison avec la saison sèche qui dure presque huit (8) mois (octobre à mai). Au cours des dix (10) dernières années (1996-2005), la température moyenne annuelle dans la région s'élève à 29,2 ${ }^{\circ} \mathrm{C}$. Le minimum est atteint en décembre et janvier avec $16,2{ }^{\circ} \mathrm{C}$ et le maximum en avril- mai avec $41,1{ }^{\circ} \mathrm{C}$ en moyenne. $\mathrm{La}$ pluviométrie est variable. Ainsi, du Nord au Sud, on trouve des zones du climat Sud Saharien (moins de $150 \mathrm{~mm}$ de pluies par an), Nord Sahélien (150 à $350 \mathrm{~mm})$ et Sud Sahélien (350 à $600 \mathrm{~mm})$. Le Sud du département de Birni N'Konni est mieux arrosé avec plus de $450 \mathrm{~mm}$, tandis que l'extrême Nord de la région (département de Tchinta) présente une moyenne annuelle des précipitations d'environ $150 \mathrm{~mm}$. Les températures varient entre $47^{\circ} \mathrm{C}$ en avril - mai et $15^{\circ} \mathrm{C}$ en décembre à janvier (moyennes respectives des maxima et minima journaliers)

La géomorphologie de la région de Tahoua se caractérise par deux grands ensembles : la zone de plateau (l'AderDoutchi-Maggia) d'une altitude moyenne comprise entre 300 et $500 \mathrm{~m}$ et un point culminant $(746 \mathrm{~m})$ localisé à la limite des départements de Keita et Abalak. Ce plateau est découpé par des vallées avec des versants de $200 \mathrm{~m}$ à l'Est et seulement $30 \mathrm{~m}$ à l'Ouest. La zone des plaines à l'Est de Madaoua, SudOuest de Konni, Ouest d'Illéla et dans le Nord se trouvent les plaines de Tamesna et d'Azaouagh.

L'agriculture constitue la principale activité des habitants de la vallée. Elle est pratiquée en saison hivernale et en saison froide. Les principales cultures pluviales pratiquées sont: le mil, le sorgho, le niébé, l'arachide et le gombo. En saison sèche, les habitants pratiquent la culture de décrue principalement la dolique dans la vallée (Figure 2) et du maraîchage autour des 
quelques creusés dans leurs jardins. L'agriculture irriguée connaît ces dernières années un regain d'intérêt et est pratiquée par l'ensemble de la population sans distinction de catégories sociales ou de sexe. La principale contrainte au développement de l'agriculture irriguée reste l'insuffisance des ressources en eaux qui ne permet pas d'emblaver l'ensemble du potentiel irrigable. Pour améliorer leur condition de vie, les habitants tentent de mettre l'accent sur le développement des cultures à valeur commerciale leur permettant de générer des revenus substantiels. L'élevage constitue la deuxième activité de la population. On distingue trois (03) types d'élevage dans la zone à savoir la transhumance, l'élevage sédentaire et l'embouche. Le cheptel est composé de bovins, ovins, caprins, camelins, équins, asins et de la volaille. En matière de santé animale, les épizooties les plus fréquentes sont: la clavelée ou variole caprine, la fièvre aphteuse et le charbon bactérien. L'alimentation des animaux est essentiellement basée sur les ressources des aires de parcours, le fourrage aérien et les résidus de cultures.

\section{Collecte des données sur la végétation}

Pour caractériser la végétation ligneuse, des unités d'échantillonnage (placette) ont été disposés de façon systématique sur des transects de 3 à $4 \mathrm{~km}$. Le long de chaque transect, des placettes de $50 \mathrm{~m} \times 50 \mathrm{~m}(2500$ $\mathrm{m}^{2}$ ) ont été disposées et orientées suivant la toposéquence c'est-à-dire du plateau vers le bas-fond jusqu'à la rencontre du plateau suivant. Cette orientation correspond à un gradient d'humidité et de profondeur du sol (Atta et al., 2010). Les paramètres dendrométriques relevés sont :
- Le diamètre du tronc à $1,30 \mathrm{~m}$ pour les arbres et à $20 \mathrm{~cm}$ pour les arbustes multicaules ;

- La hauteur à 1,30 m pour les arbres ;

- Le diamètre moyen du houppier ;

- Et les nombres des tiges.

Ces données ont été collectées dans le but de déterminer :

la diversité systématique exprimée par le nombre d'espèces (ou richesse spécifique), de genres et de familles, les types biologiques dont la détermination est réalisée suivant la classification de Raunkiaer (1916), le types phytogéographiques et l'indice de diversité $\alpha$ qui évalue les relations existant entre les espèces au sein d'une même communauté. Cet indice renseigne sur la diversité des espèces au sein d'un habitat ou d'une communauté. Il est d'autant plus élevé qu'un grand nombre d'espèces participe au recouvrement. Il s'exprime en bits par individus. D'une manière générale, sa valeur varie 0 à 5 voir un peu plus de 5 bits ou davantage pour de très grands échantillons. La diversité $\alpha$ est calculée en utilisant l'indice de Shannon-Weaver selon la formule suivante :

$$
\begin{gathered}
\mathbf{H}=-\sum_{\boldsymbol{i}=\mathbf{1}}^{\mathbf{s}} p i \log 2 p i \\
\boldsymbol{P i}=\boldsymbol{n i} / \boldsymbol{S}
\end{gathered}
$$

où $\mathrm{pi}=\mathrm{ni} / \mathrm{S}, \mathrm{S}=$ nombre total d'espèces;

$\mathrm{ni}=$ fréquence de groupement.

Ces données ont permis également de calculer l'équitabilité de Piélou $\mathrm{R}$ à partir de l'indice de Shannon-Weaver :

$$
\mathrm{R}=\mathrm{H}^{\prime} / \log 2(\mathrm{~S}) \quad \mathrm{R} \in[0-1]
$$

Si $R \in[0$ - 0,6] alors l'équitabilité de Pielou est faible (phénomène de dominance existant dans la communauté).

Si $R \in[0,7-0,8[$ alors l'équitabilité de Pielou est moyenne. 
Si $R \in[0,8-1]$ alors l'équitabilité de Pielou est élevée (absence de dominance dans la communauté).

Les relevés phytosociologiques ont été effectués suivant la méthode BrauneBlanquet. Cette méthode consiste à affecter à chaque espèce ligneuse présente dans la placette un coefficient d'abondancedominance. L'échelle de Braune-Blanquet se présente comme suit :

5 : espèce recouvrant plus de $75 \%$ de la surface du relevé ;

4 : espèce recouvrant entre $50 \%$ et $75 \%$ de la surface du relevé ;

3 : espèce recouvrant entre $25 \%$ et $50 \%$ de la surface du relevé ;

2 : espèce recouvrant entre $5 \%$ et $25 \%$ du relevé ;

1 : espèce abondante à peu abondante et recouvrant moins de $5 \%$ de la surface du relevé ;

+ : espèce rare et recouvrant moins de $5 \%$ de la surface du relevé.

Les recouvrements moyens (RM) correspondant à chaque classe d'abondance-dominance sont :

5 : espèce à recouvrement moyen de $87,5 \%$;

4 : espèce à recouvrement moyen de $62,5 \%$;

3 : espèce à recouvrement moyen de $37,5 \%$;

2 : espèce à recouvrement moyen de $15 \%$;

1 : espèce à recouvrement moyen de $3 \%$.

+ : espèce à recouvrement moyen de $0,5 \%$.

Cette technique a été utilisée par plusieurs auteurs dans l'étude de la végétation en Afrique tropicale (Mahamane, 2005 ; Idrissa, 2011).

\section{Analyses des données}

\section{Données dendrométriques}

Les données dendrométriques ont été analysées à partir du logiciel Excel, ce qui a permis de déterminer les structures horizontales et verticales du peuplement ligneux.

\section{Données phytosociologiques}

Les données phytosociologiques collectées ont été analysées à l'aide du Logiciel PC-Ord version 5 (McCune and Mefford, 2006). Ainsi, la matrice des données présence absence, constituée par les relevés et les espèces, a été soumise à une analyse factorielle des correspondances redressée (DCA : Detrended Correspondence Analysis.

\section{RESULTATS}

\section{Caractéristiques de la végétation}

Composition floristique

Dans l'ensemble, soixante (60) relevés ont été effectués avec une richesse spécifique (S) de vingt-deux (22) espèces réparties en neuf (09) familles. Le Tableau 1 donne la liste des espèces et de leurs familles. Il ressort de ce tableau que les familles des FabaceaeMimosoideae sont les plus importantes avec 7 espèces (soit 31,81\%), ensuite viennent les Combretaceae avec 4 espèces (soit 18,18\%), puis les Capparaceae avec 3 espèces (soit 13,63\%). Les Fabaceae-Cesalpinoideae et les Asclepiadaceae représentent chacune 2 espèces (soit 9,09\%), et enfin les Rhamnaceae, les Anacardiaceae, les Rubiacea et le Balanitaceae avec chacune 1 espèce (soit $4,54 \%)$.

\section{Ordination des relevés}

La matrice abondance-dominance composée de 60 relevés et 22 espèces a été soumise à une analyse factorielle des correspondances, ce qui a permis de révéler un seuil de similarité de $18,75 \%$, d'identifier trois groupements végétaux qui sont $\mathrm{G}_{\mathrm{I}}, \mathrm{G}_{\mathrm{II}}$ et $\mathrm{G}_{\text {III }}$ comme l'indique le dendrogramme de la Figure 2 ci-dessous. Le groupement $\mathrm{G}_{\mathrm{I}}$ est 
constitué des relevés effectués sur les plateaux. Ces plateaux sont situés en altitude et sont faiblement utilisés pour l'agriculture mais surtout comme aires de pâturage. Ces sols sont pauvres en matières organiques et ont un $\mathrm{pH}$ acide. Ils sont de type ferrugineux tropical. Ces plateaux dégradés témoignent de l'action érosive très forte conduisant à une perte des terres énorme. Aujourd'hui, avec les actions de récupération des terres, ces plateaux sont revégétalisés. Ils sont ainsi dominés surtout par des Acacia utilisés pour la récupération des terres.

Le groupement $\mathrm{G}_{\mathrm{II}}$ est constitué des relevés effectués sur des sols des bas-fonds ou Fadama. Ils sont très riches en matières organiques avec une végétation très abondante. Ce sont des vallées périodiquement inondées pendant la saison pluvieuse et qui disposent des sols de types argileux, tandis que les lits de Koris sont sableux. Ces sols disposent d'un haut rendement pour les cultures pluviales et maraîchères et sont surtout colonisés par des espèces végétales telles que Ziziphus mauritiana, Faidherbia albida, Acacia nilotica, Acacia tortilis, Piliostigma reticulatum, Guiera senegalensis Acacia seal.

Le groupement $\mathrm{G}_{\mathrm{III}}$ est constitué des relevés qui évoluent sur des sols de glacis ou Fako, le plus souvent incultes sur lesquels les paysans réalisent des travaux de récupération des terres. Ils sont pauvres en matières organiques et leur aptitude agricole est actuellement dégradée. La couverture végétale $\mathrm{y}$ est très faible, entraînant une activité érosive très importante.

\section{Les types biologiques}

La Figure 4 montre que la flore du bassin versant de la Maggia est dominée par des microphanérophytes qui en représentent $95,45 \%$, suivis des nanophanerophytes avec $4,5 \%$.

\section{Les types phytogéographiques}

La Figure 5 représente les types phytogéographiques. Il ressort de l'analyse de cette figure que les espèces SoudanoZambéziennes (SZ) sont les plus dominantes $(50 \%)$, suivies des espèces SoudanoZambéziennes-Saharo-Sindiènnes (SZ-Sah.S) (40,9\%). Les Guinéo-Congolaises-SoudanoZambéziennes-Saharo-Sindienne sont minoritaires et ne constituent que $9,09 \%$.

\section{Structure démographique du peuplement Structure en hauteur}

L'analyse de la structure verticale du peuplement ligneux (Figure 6) montre que la flore du bassin versant de la Maggia est dominée par des individus jeunes ayant une hauteur comprise entre [0-5m [. Les individus de hauteur supérieure à $7 \mathrm{~m}$ et inférieure à 10 $\mathrm{m}$ sont très peu nombreux et ceux de hauteur supérieure à $10 \mathrm{~m}$, sont presque absents dans cette zone.

\section{Structure en diamètre}

La Figure 7 montre la structure horizontale du peuplement ligneux. L'analyse des histogrammes de ce peuplement montre que la flore du Bassin versant de la Maggia est surtout dominée par des individus de faible diamètre. Les individus de gros diamètre sont très peu nombreux et plus le diamètre augment, plus le nombre des individus diminue, voire deviennent complètement absent. Dans cette zone, les quelques rares individus de gros diamètre représentent surtout ceux conservés dans les champs par des paysans pour des raisons socioéconomiques, culturelles, voire religieuses. $\mathrm{Ce}$ sont surtout des espèces comme Acacia albida, Pilostigma reticulatum, Acacia tortilis, Combretum glutinosum. 


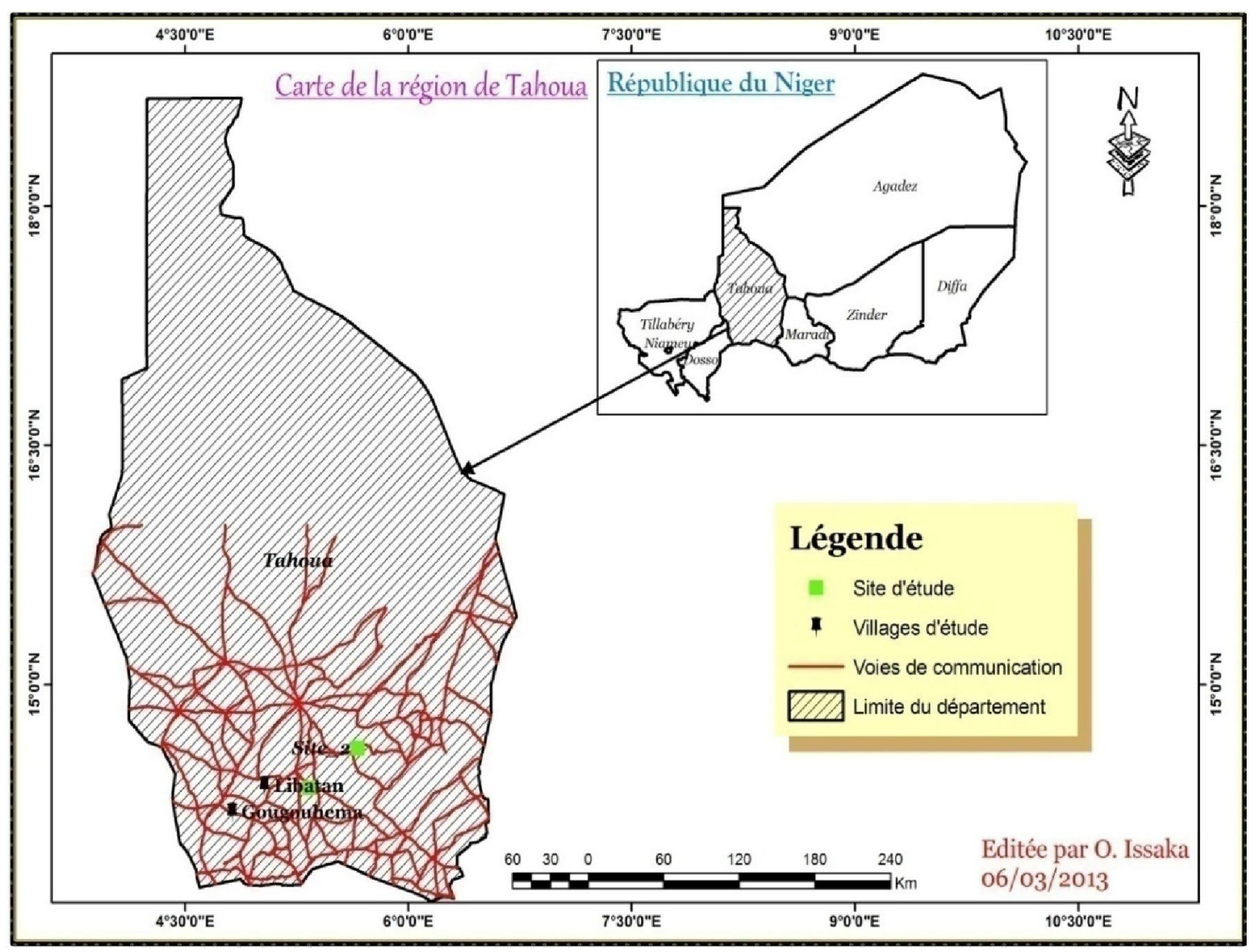

Figure 1 : Carte de la limite de la région de Tahoua (GARBA Amadou, 2013).
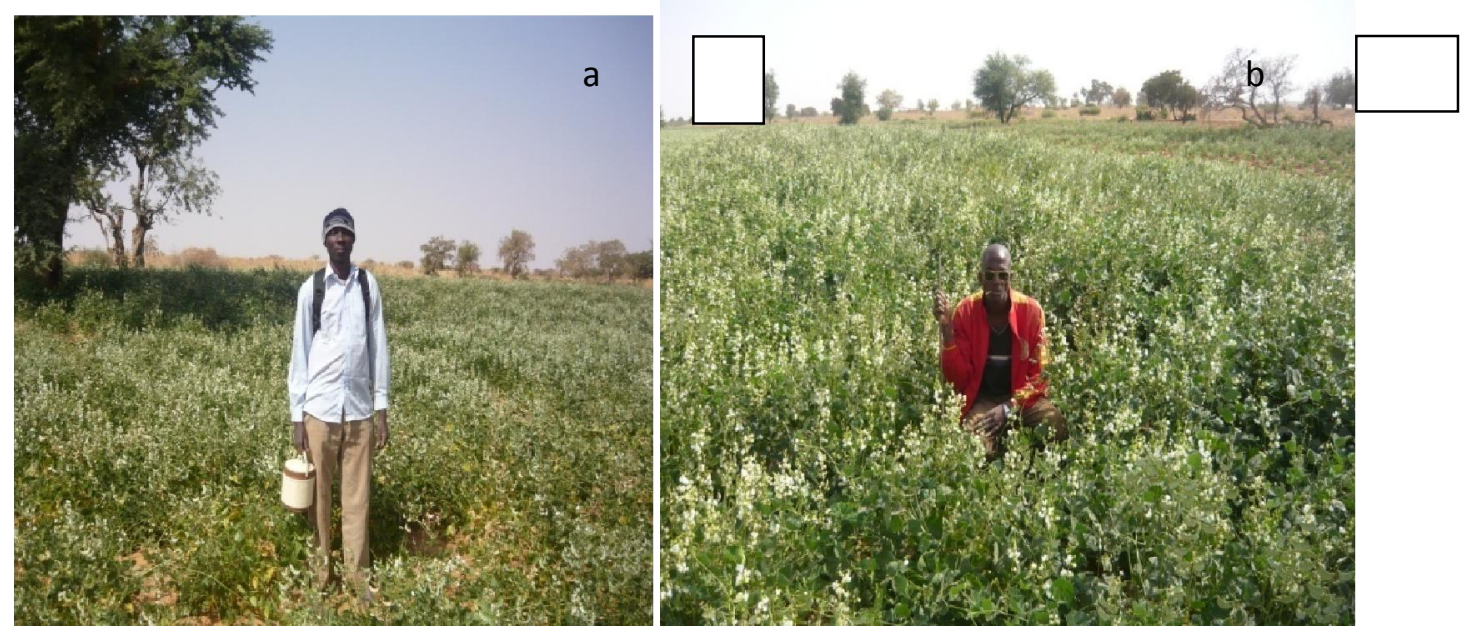

Figure 2: Culture de dolique dans la vallée de la Maggia à Gougouhema et Libatan pendant la décrue. a) Libatan ; b) Gougouhema. 
Tableau 1 : Nombre d'espèces recensées dans le bassin versant de la Maggia avec leurs familles.

\begin{tabular}{lccc}
\hline Espèces & Familles & Effectifs & Individus/ha \\
\hline Guiera senegalensis & Combretaceae & 39 & 2,6 \\
Acacia senegal & Fabaceae-Mimosaceae & 16 & 1,1 \\
Acacia seyal & Fabaceae-Mimosaceae & 19 & 1,3 \\
Combretum micrantum & Combretaceae & 4 & 0,3 \\
Acacia nilotica & Fabaceae-Mimosaceae & 21 & 1,4 \\
Bossia angustifolia & Caparaceae & 3 & 0,2 \\
Bossia Senegal & Caparaceae & 33 & 2,2 \\
Bauhinia rufescens & Fabaceae-Cesalpinoideae & 2 & 0,1 \\
Combretum glutinosum & Combretaceae & 89 & 5,9 \\
piliostigma reticulatum & Fabaceae-Cesalpinoideae & 41 & 2,7 \\
Maerua crassifolia & Caparaceae & 2 & 0,1 \\
Sclerocaria birrea & Anacardiaceae & 10 & 0,6 \\
Ziziphus mauritiana & Rhamnaceae & 17 & 1,1 \\
Acacia radiana & Fabaceae-Mimosaceae & 3 & 0,2 \\
Balanites aegyptiaca & Balanitaceae & 28 & 1,9 \\
Calotropis procera & Asclepiadaceae & 1 & 0,1 \\
Faiderbia albid & Fabaceae-Mimosaceae & 1 & 0,1 \\
Prosopis Africana & Fabaceae-Mimosaceae & 1 & 0,1 \\
Feretia apondonthera & Rubiaceae & 2 & 0,1 \\
Terminalia avicennoides & Combretaceae & 9 & 0,6 \\
Albizia chevalieri & Fabaceae-Mimosaceae & 2 & 0,1 \\
Leptadenia hastata & Asclepiadaceae & 4 & 0,3 \\
22 & & $\mathbf{2 3 , 6}$ \\
\hline
\end{tabular}

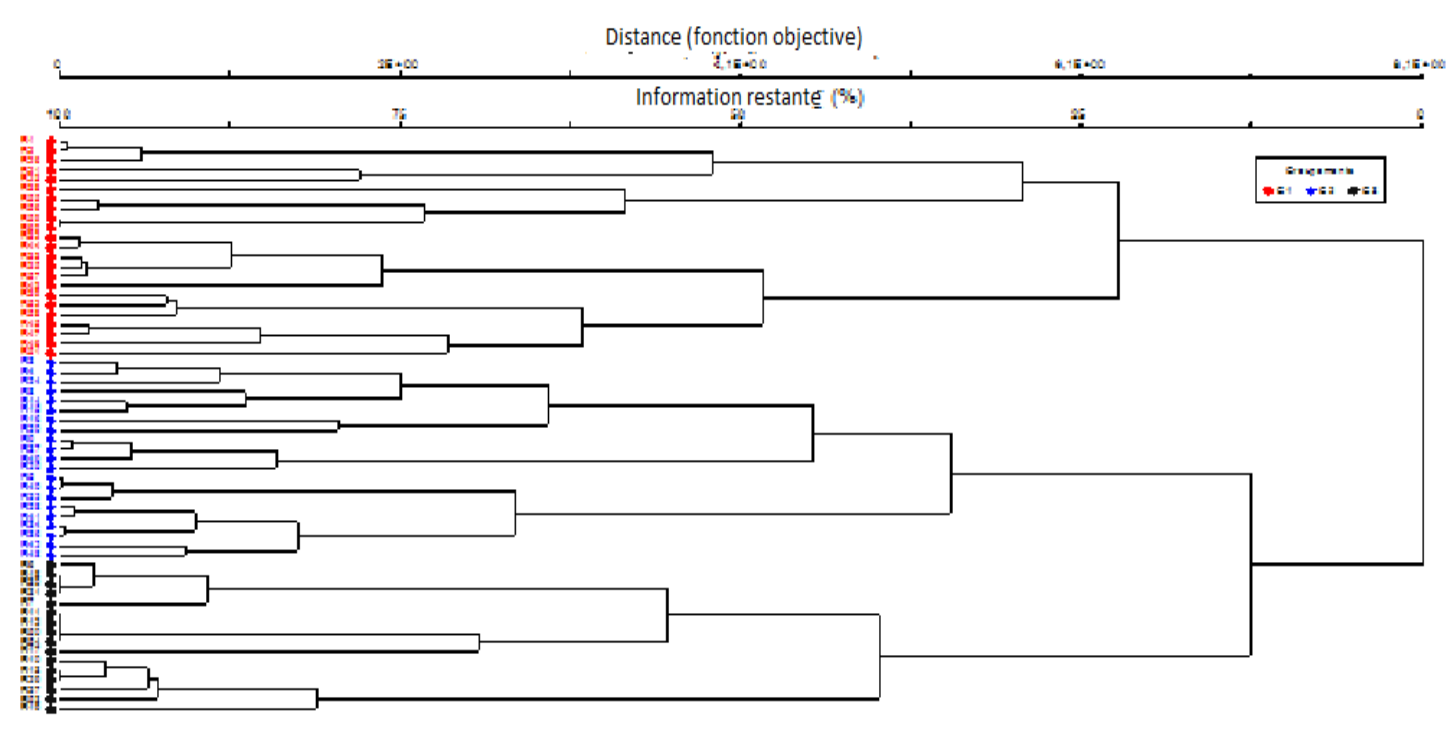

Figure 3: Classification ascendante des groupements végétaux du bassin versant de la Maggia. 


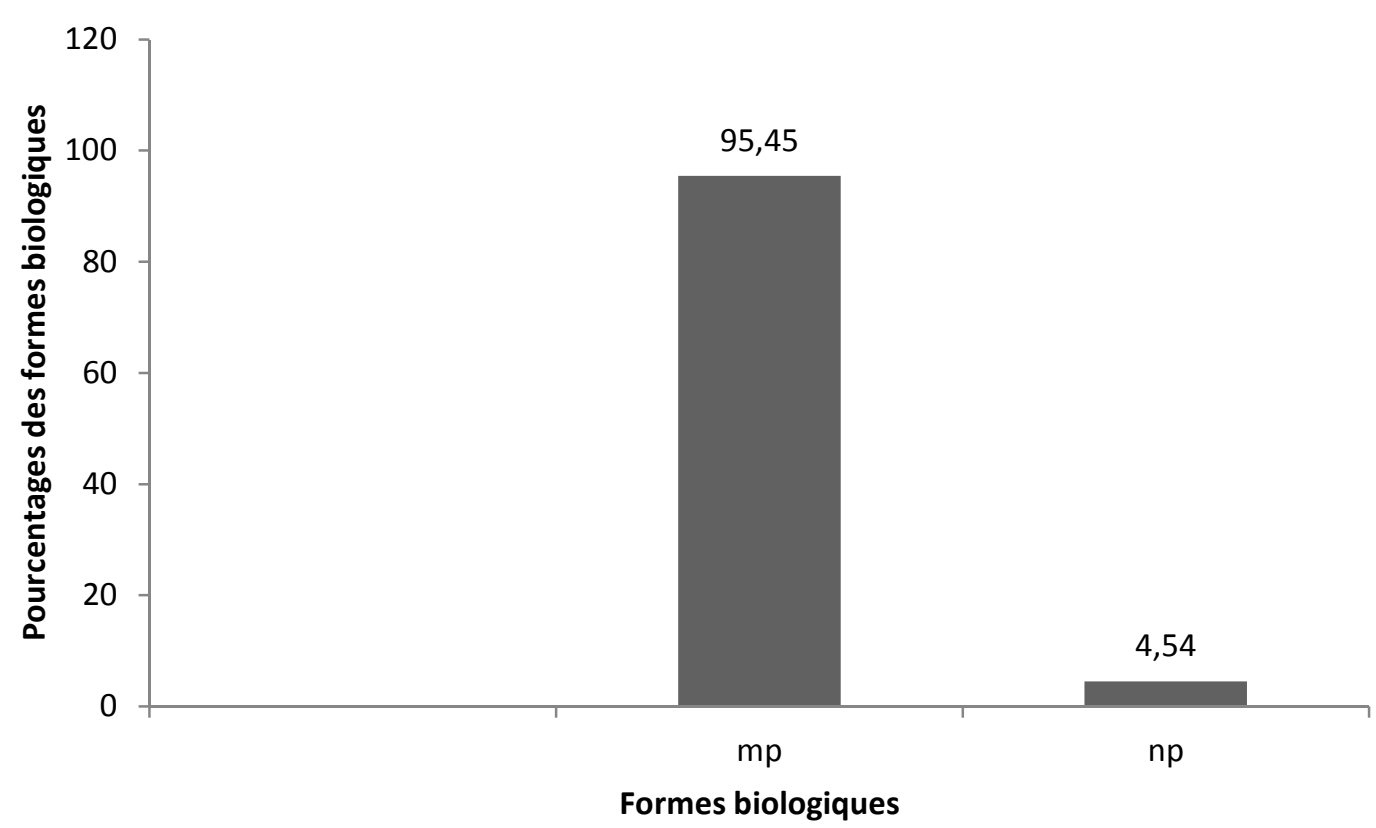

Figure 4 : Types biologiques. $\mathrm{Mp}=$ microphanérophytes $; \mathrm{Np}=$ nanophanerophytes .

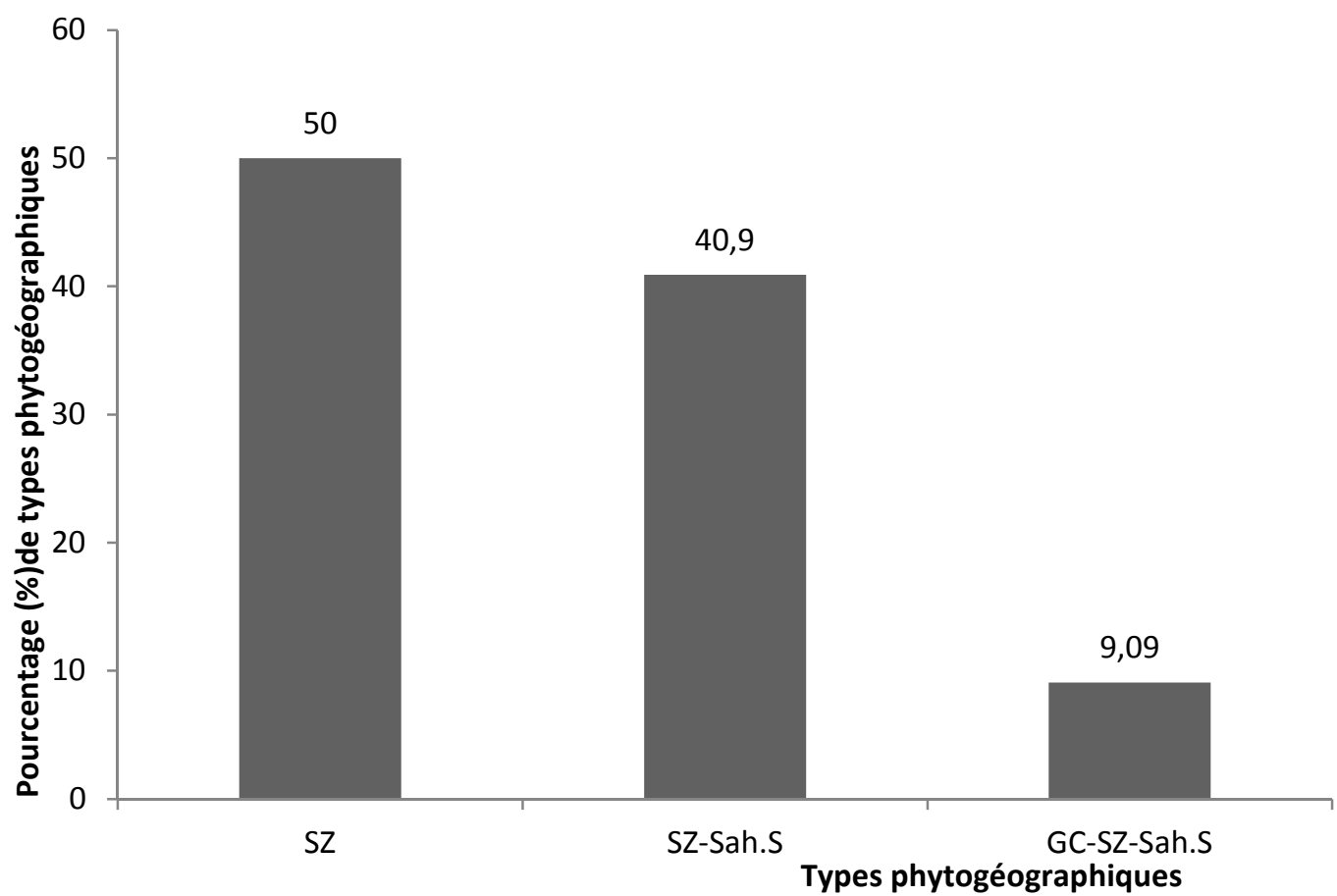

Figure 5 : Types phytogéographiques. 


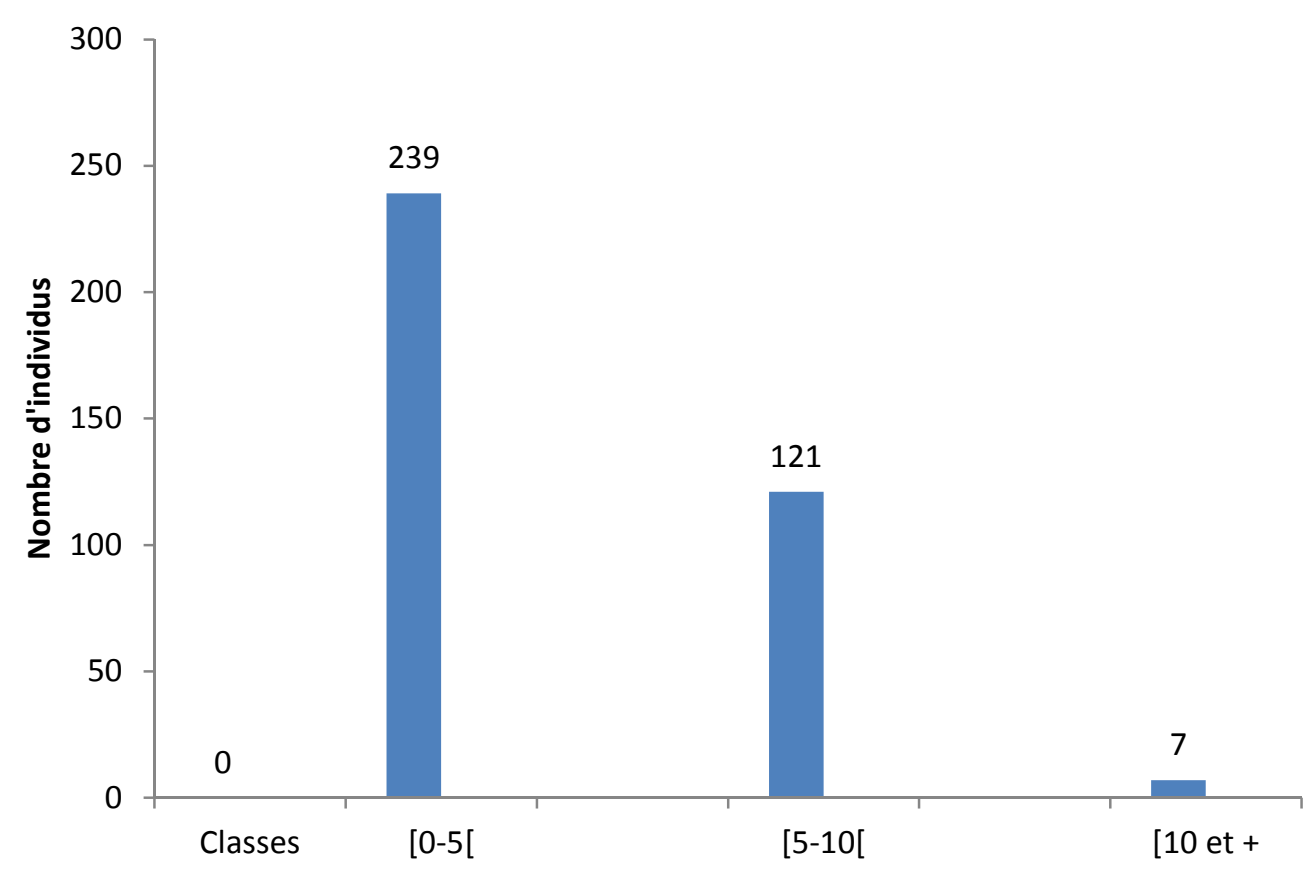

Figure 6 : Classes en hauteur (m) du peuplement ligneux du bassin versant de la Maggia.

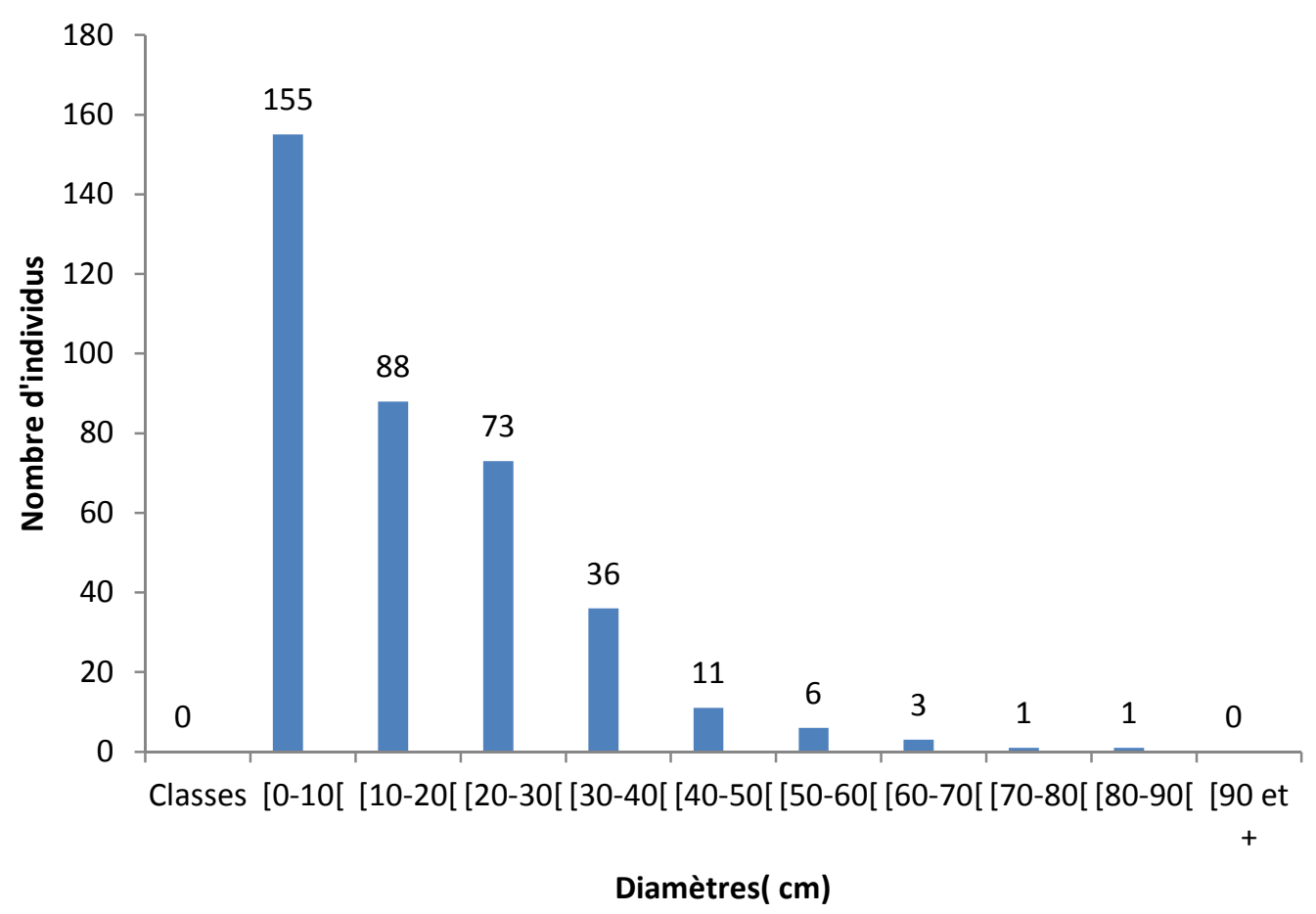

Figure 7 : Classe en diamètre $(\mathrm{cm})$ du peuplement ligneux du bassin versant de la Maggia. 


\section{DISCUSSION}

Etat actuel du peuplement ligneux dans la zone

\section{Richesse spécifique}

$\mathrm{La}$ richesse spécifique du bassin versant de la Maggia est de 22 espèces réparties en neuf (09) familles. Les résultats de cette étude ont démontré que la flore de la végétation de cette zone est surtout dominée par des Fabaceae-Mimosoideae, suivies des Combretaceae. Ces résultats corroborent ceux de Djibo (2012) qui a évalué la diversité des peuplements ligneux des champs du bassin versant de Badaguichiri dans la région de Tahoua, où il a trouvé une richesse spécifique de 24 espèces. Les indices de diversité de Schannon-Weaver déterminés à travers le recouvrement des espèces montrent que les groupements étudiés sont moins diversifiés. Il est de 0,61 bits pour $G_{1}, 0,91$ bits pour $G_{2}$ et 0,34 bits pour $\mathrm{G}_{3}$. Cette faible diversité des groupements est surtout due aux facteurs anthropiques car au Niger, la problématique de la gestion des ressources naturelles se caractérise par un déséquilibre entre les besoins accrus des populations en croissance rapide $(3,3 \%)$ et la recherche d'une amélioration générale de leur condition de vie. Ainsi, les formations forestières se dégradent à des rythmes inquiétants au profit de l'agriculture et de l'élevage pratiqués de manière extensive (Boubé, 2010). L'explosion démographique de la population entraîne une augmentation de l'intensité et des fréquences de prélèvement sur les ressources végétales et c'est la pauvreté des populations qui ellemême résulte de la baisse des rendements agricole, qui oblige les ménages à se comporter ainsi (Barmo, 2008). Ces actions anthropiques qui sont la base de la perte de la biodiversité végétale constituent un impact majeur pour l'équilibre des écosystèmes naturels. La réduction de la biodiversité biologique d'un écosystème peut diminuer sa flexibilité face à la perturbation et peut augmenter sa fragilité face aux maladies; ce qui menace sa stabilité et son intégrité (PNUD, 2002). La Figure 8 témoigne des pressions anthropiques sur les espèces ligneuses. Ces pratiques qui sont fréquentes dans la zone concourent fortement à une érosion génétique des espèces ligneuses fruitières qui contribuent à la résilience des populations pendant les périodes de soudure qui sont vécues généralement par les populations comme une crise silencieuse. Les populations développent alors des stratégies qui leur sont propres en s'adaptant à leur environnement par une exploitation des ressources végétales alimentaires, (Janin, 2004 ; Ouedraogo, 2006; Daniabla et al., 2012).

\section{Les formes biologiques}

L'analyse du comportement adaptatif des espèces montre que ce sont les microphanérophytes qui dominent dans cette zone. Ces résultats sont conformes à ceux trouvés par Abdou (2008) qui a évalué la biodiversité végétale des milieux de culture du souchet (Cyperus esculentus L.)

\section{Les formes phytogéographiques}

Dans la répartition phytogéographique, les affinités chorologiques SoudannoZambéziennes (SZ) dominent dans leur grande majorité suivies des espèces SoudanoZambéziennes-Saharo-Sindiennes (SZ-Sah.S).

\section{Structures en hauteur et en diamètre du peuplement ligneux}

La répartition par classes de diamètre et de hauteur montre respectivement une faible densité des individus de gros diamètre et de hauteur supérieure à $7 \mathrm{~m}$. Le nombre des pieds à l'hectare du peuplement est de 25 . Cela démontre également la faible densité des espèces dans la zone. Cette diminution des densités est liée à l'action anthropique (feu de brousse, coupe abusive) et aux épisodes de 
sécheresse et de changement climatique qu'a connu et connait encore le Sahel (Lawanou et al., 2005 ; Gonzalez et al., 2012 ; Bakhoum et al., 2012; Ouango et al., 2015). A ces facteurs, on peut aussi ajouter l'impact des surpâturages des animaux qui peut contribuer à la disparition des certaines espèces végétales dans le milieu. L'analyse des histogrammes des classes de diamètre et de hauteur montre également que la flore du bassin versant de la Maggia est dominée par des individus jeunes. Cette prédominance des individus jeunes est due non seulement aux actions de la régénération naturelle assistée que pratique les paysans dans cette zone et qui prend de plus en plus d'ampleur mais aussi à travers les résultats des actions de récupération des terres dégradées comme les banquettes sur plateaux érodés et les demi-lunes sur les glacis. Les paysans ayant pris connaissance de l'utilité des arbres (fertilisation, nutrition et alimentation), et des conséquences environnementaux auxquels ils sont exposés (vents, sécheresse, famine) ont contribué au reverdissement par la pratique de la régénération naturelle assistée (RNA) (Ouango et al., 2015). Cela peut être dû à plusieurs paramètres liés notamment aux pressions anthropiques, à la nature du sol et aux effets des variabilités climatiques, aux surpâturages dans la zone, aux feux de brousse, aux pratiques agricoles non adaptées etc. Cette faible densité des individus témoigne de la présence d'un milieu fortement anthropisé. A ces facteurs anthropiques s'ajoutent les facteurs climatiques car la géomorphologie de cette zone est surtout caractérisée par des vastes plateaux dégradées et des glacis qui concourent fortement aux actions érosives. L'érosion contribue non seulement à la dégradation des terres, mais aussi à appauvrir les sols en semence pour la régénération naturelle et la diversité génétiques des espèces qui est un facteur important dans le processus d'adaptation des espèces face aux changements climatiques.

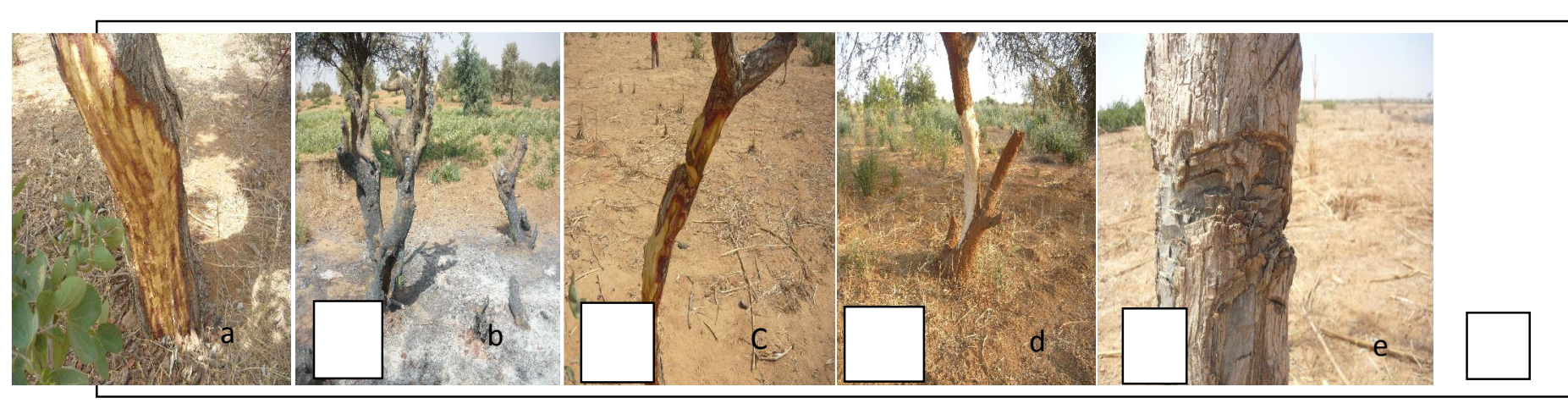

Figure 8 : Pression anthropique sur les espèces ligneuses présentes dans le bassin versant de la Maggia. (a) Ecorçage de Piliostigma reticulatum, (b) destruction de Pilostigma reticulatum par le feu dans la vallée de la Maggia à Gougouhema, (c) : coupe et écorçage de Terminalia avicennioides, (e) : coupe de Faidherbia albida, (d) : écorçage de Acacia tortilis. 


\section{Conclusion}

La dégradation des ressources naturelles dans la zone sahélienne est due essentiellement à de mauvais modes de gestion, à leur surexploitation et aux sécheresses répétitives issues des différentes crises climatiques. Les résultats de la présente étude ont permis de déterminer la composition floristique de ce bassin versant et de comprendre les différentes menaces qui pèsent sur les différentes espèces ligneuses de cette région. Les principales causes de ces menaces sont surtout liées à la croissance démographique, à la pauvreté des populations et à l'aridité du climat. Pour sauvegarder cette biodiversité végétale, il est indispensable de poursuivre plusieurs actions à la fois dont notamment la sensibilisation des populations de cette localité pour une prise de conscience sur la nécessité de conserver les ressources naturelles sur lesquelles repose leur bien-être, afin d'une part de lutter contre la désertification, source de crise alimentaire au sahel et d'autre part, d'entreprendre des actions de reboisements et d'élaboration des plans d'aménagements. Pour la réussite de ces programmes, il s'avère nécessaire de lutter contre la dégradation de l'environnement, de développer une approche intégrée et participative de toutes les parties prenantes, d'essayer de répondre aux attentes des populations locales et de satisfaire leurs besoins prioritaires, d'où l'intérêt de concilier les besoins du développement et les impératifs de protection de l'environnement.

\section{CONFLIT D'INTERETS}

Il n'y a pas de conflit d'intérêt entre les auteurs de cet article.

\section{CONTRIBUTIONS DES AUTEURS}

AG est l'investigateur principal ; ITD et LA ont contribué à la rédaction du manuscrit; AM a conçu le protocole de recherche et a supervisé le travail.

\section{REMERCIEMENTS}

$\mathrm{Au}$ terme de cette étude nous remercions Dr. Ir. Abdourhamane Hamidou
Yero, Mr BOLA Moussa coordonateur national $\mathrm{du}$ Projet PRODEX et $\mathrm{Mr}$ ADARKAS Oumorou point focal/PRODEX qui ont tous apporté leur soutien matériel et financier pour la réussite de ce travail. Nous remercions également les chefs des villages de Gougouheme et Libatan, ainsi que leurs populations qui ont brillé par leur hospitalité durant notre séjour.

\section{REFERENCES}

Abdou A. 2008. Biodiversité végétale des milieux de cultures du souchet (Cyperus esculentus L.) dans la région de Maradi (Niger), Université Abdou moumouni de Niamey. Mémoire de D.E.A. Biologie appliquée, $110 \mathrm{p}$.

Abdourahamane HY. 2016. Etude floristique, écologique, et ethnobotanique des forêts classées de dan-kada dodo dans gado au Niger. Thèse de Doctorat en sciences de l'Environnement. Université Dan Dicko Dankoulodo de Maradi, 209 P.

Barmo S. 2008. Analyse socio-économique de l'exploitation des ressources végétales de la réserve Totale de Faune de Tamou, République du Niger. Mémoire de DEA Biologie appliqué, $88 \mathrm{p}$.

Bakoum C, Agbangba EC, Ndour B. 2012. Natural Regeneration of Tree in Arid and Semi-Arid Zones in West Africa. Journal of Asian Scientific Research, 2(12) 820-834.

Djibo S. 2012. Diversité des peuplements ligneux des champs du Bassin versant de Badaguichiri (Tahoua/Niger). Mémoire de master 2, présenté pour l'obtention du diplôme en gestion des ressources naturelles et de la biodiversité, 68p.

Guinko S. 1984. Végétation de Haute Volta. Thèse de Doctorat ès. Sci. Nat., Université de Bordeaux III, 318P.

Gonzalez P, Tucker CJ, SY H. 2012. Tree density and species decline in the African Sahel attributable to climate. Journal of Arid Environments, 78: 5564.

Hountondji YC. 2008. Dynamique environnementale en zones sahélienne et 
soudanienne de l'Afrique de l'Ouest : Analyse des modifications et évaluation de la dégradation du couvert végétal. Thèse de Doctorat en Sciences de l'Environnement. Université de LiègeBelgique, $153 \mathrm{p}$.

Idrissa S. 2012. Groupements végétaux pâturés des parcours de la région de Zinder et stratégies d'exploitation développées par les éleveurs Uda'en. Thèse présentée pour obtenir le grade de docteur en sciences biologiques appliquées spécialité : Écologie végétale et Pastoralisme à l'Université Abdou Moumouni de Niamey, $229 \mathrm{P}$.

Janin P. 2000. La gestion spatio-temporelle de la soudure alimentaire dans le sahel burkinabé. Revue Tiers-Monde 45: 90933.

Larwanou M, Saadou M. 2005. Biodiversity of ligneous species in semi-arid to arid zones of southwestern Niger according to anthropogenic and natural factors. Agriculture, Ecosystems and Environment, 105: 267-271

Mahamane A. 2001. Usages des terres et évolutions végétales dans le département de Maradi. Drylands Research, 27: 43 p.

Mahamane A. 2005. Etudes floristique, phytosociologique et phytogéographique de la végétation du Parc W Niger. Thèse présentée à l'Université de bruxelles pour obtenir le titre de Docteur, 516P.

Ouango MS, Korodjouma O, Jennie B, Issa O, Line G, Elin E, Nabsanna PZ. 2015.
Etats des écosystèmes sahéliens: reverdissement, perte de la diversité et qualité des sols: Afrique SCIENCE, 11(5): 433 - 446.

Oumorou M. 2000. Etudes écologique, floristique, phytogéographique et phytosociologique des inselbergs du Bénin. Thèse de doctorat, Université Libre de Bruxelles, Faculté des Sciences, Laboratoire de Botanique Systématique, $210 \mathrm{p}$.

OECD. 2012. Perspectives de l'Environnement de l'OCDE à l'Horizon 2050. Les Conséquences de l'Inaction. OECD: Paris.

Ouédraogo FC. 2006. La vulnérabilité alimentaire au Burkina Faso Ouagadougou (Burkina Faso): L'Harmattan.

PNUD. 2002. Ressources Mondiales 20002001. PNUD: Washington, USA, ESKA ; 389.

Reij C, Tappan G, Smale S. 2009. ReGreening the Sahel Farmer-led innovation in Burkina Faso and Niger in Agroenvironmental Transformation in the Sahel: Another kind of "Green Revolution". In International Food Policy Research Institute; 53-58.

Thiombiano DNE, Lamien N, Dibong DS, Boussim IJ, Belem B. 2012. Le rôle des espèces ligneuses dans la gestion de la soudure alimentaire au Burkina Faso. Secheresse, 23: 86-93. doi: $10.1684 / \mathrm{sec} .2012 .0341$ 